\title{
Correction to: Acquisition of Stop Consonants in Mandarin-Japanese Early Bilingual Children
}

\author{
Makiko Tanaka ${ }^{1} \cdot$ Li-Mei Chen ${ }^{2} \cdot$ Chung-Jen Hsu $^{3}$ \\ Published online: 23 October 2018 \\ ○) Springer Science+Business Media, LLC, part of Springer Nature 2018
}

\section{Correction to: Journal of Psycholinguistic Research https://doi.org/10.1007/s10936-018-9604-5}

The original version of the article unfortunately contained an error in Acknowledgement section. The name of the first person acknowledged in that section should be Dr. Mary Beckman.

The original article can be found online at https://doi.org/10.1007/s10936-018-9604-5.

$凶 \quad$ Makiko Tanaka

makiko_t@hotmail.com

1 Department of Applied Japanese, Southern Taiwan University of Science and Technology, Tainan, Taiwan

2 Department of Foreign Languages and Linguistics, National Cheng Kung University, Tainan, Taiwan

3 Department of Counseling, National Chiayi University, Chiayi, Taiwan 